\title{
Cosmological Particle Production in Quantum Gravity
}

\author{
Yaser Tavakoli ${ }^{1,2}$ (D) \\ 1 Department of Physics, University of Guilan, Namjoo Blv., Rasht 41335-1914, Iran; yaser.tavakoli@guilan.ac.ir \\ 2 School of Astronomy, Institute for Research in Fundamental Sciences (IPM), Tehran 19395-5531, Iran
}

\section{check for} updates

Citation: Tavakoli, Y. Cosmological Particle Production in Quantum Gravity. Universe 2021, 7, 258. https:// doi.org/10.3390/universe7080258

Academic Editor: Jaime Haro Cases

Received: 16 June 2021

Accepted: 20 July 2021

Published: 22 July 2021

Publisher's Note: MDPI stays neutral with regard to jurisdictional claims in published maps and institutional affiliations.

Copyright: (C) 2021 by the authors. Licensee MDPI, Basel, Switzerland. This article is an open access article distributed under the terms and conditions of the Creative Commons Attribution (CC BY) license (https:// creativecommons.org/licenses/by/ $4.0 /)$.

\begin{abstract}
Quantum theory of a test field on a quantum cosmological spacetime may be viewed as a theory of the test field on an emergent classical background. In such a case, the resulting dressed metric for the field propagation is a function of the quantum fluctuations of the original geometry. When the backreaction is negligible, massive modes can experience an anisotropic Bianchi type I background. The field modes propagating on such a quantum-gravity-induced spacetime can then unveil interesting phenomenological consequences of the super-Planckian scales, such as gravitational particle production. The aim of this paper is to address the issue of gravitational particle production associated with the massive modes in such an anisotropic dressed spacetime. By imposing a suitable adiabatic condition on the vacuum state and computing the energy density of the created particles, the significance of the particle production on the dynamics of the universe in Planck era is discussed.
\end{abstract}

Keywords: loop quantum cosmology; quantum fields in curved spacetime

\section{Introduction}

Observation of cosmic microwave background (CMB) implies that the classical universe is homogeneous and isotropic on scales larger than 250 million light years. Based on the standard $\Lambda C D M$ model of cosmology, the Friedmann-Lemaître-Robertson-Walker (FLRW) solution of Einstein's field equations provides a suitable explanation of such near perfect isotropy of the $\mathrm{CMB}$ and other astrophysical observables. Nevertheless, there is not yet a decisive answer to the question of whether or not the quantum structure of the universe in the super-Planckian regime has the same symmetry as observed in the CMB and how such structure can be traced in the observational data.

According to the standard model of cosmology, the structure formation at large scales are described via (inhomogeneous) perturbations at smaller scales in the early universe. Quantum field theory in classical, curved spacetime provides a good approximate description of such phenomena in a regime where the quantum effects of gravity are negligible [1,2]. Within such framework, the issue of the gravitational particle production induced by the time-dependent background in an expanding universe and its backreaction effect is of significant importance [3,4]. Nevertheless, when tracing further back in time, where the curvature of the universe reaches the Planck scales, the quantum effects of gravity become important. This might lead to additional phenomena that are expected to be important when exploring the dynamics of the early universe.

Loop quantum cosmology (LQC) is a promising candidate to investigate the quantum gravity effects in Planckian regime [5]. It follows the quantization scheme of loop quantum gravity (LQG), which is a background independent, non-perturbative approach to the quantization of general relativity [6-8]. This approach has provided a number of concrete results: the classical big bang singularity is resolved and is replaced by a quantum bounce [9-12]; the standard theory of cosmological perturbations has been extended to a self-consistent theory from the bounce in the super-Planckian regime to the onset of slow-roll inflation [13-17]. Further phenomenological consequences and observational predictions of LQC can be found, e.g., in Refs. [17-29], respectively. 
The cosmological quantum backgrounds in LQC establish a fruitful ground to explore the quantum theory of inhomogeneous fields propagating on it. In a dressed metric approach, when the full quantum Hamiltonian constraint of the gravity-matter system is solved, an effectively dressed geometry emerges for the field modes. Depending on whether the field is massless or massive, various spacetime metrics can raise for the dressed background geometry. The emergent metric components, if the backreaction between the fields and the geometry is discarded, depend, generically, on the fluctuations of the original quantum metric operator only. For massive modes, quantum gravity effects may induce a small deviation from the initial isotropy $[19,24]$. If the backreaction is considered, other properties, such as emerging a mode-dependent background, can raise, which leads to the violation of the local Lorentz symmetry [18,20]. Even in the absence of the backreaction between the states of the gravity and matter fields, a backreaction effect may arise due to the gravitational particle production, which can challenge the validity of the test field approximations employed in the dressed metric scenario [22].

Motivated from the above paragraphs, our purpose in this article is to address some phenomenological issues associated with the massive test field propagating on a quantum cosmological spacetime. In particular, we will consider a scenario in which an anisotropic dressed geometry emerges for the massive modes due to quantum gravity effects and then revisit the occurrence of the gravitational particle production on such an anisotropic dressed background. Such effects will have important consequences in the super-Planckian regime and at the onset of the classical inflationary epoch. We thus organize this paper as follows. In Section 2, we will consider the propagation of a massive test field on a quantized FLRW spacetime. We will derive an effective evolution equation for the field and obtain a suitable anisotropic dressed background for the field propagation. In Section 3, we will study the theory of the quantum field by considering the many infinite modes on the emergent anisotropic spacetime. We will then explore the problem of the gravitational particle production by choosing a convenient, adiabatic vacuum state in the super-Planckian regime. Finally, in Section 5, we will present the conclusion and discussion of our work.

\section{Quantum Fields on a Quantum FLRW Spacetime}

In this section, we study the quantum theory of a massive scalar field on a quantum FLRW background. Firstly, we will analyze the quantum theory of a scalar field in the classical Friedmann universe. Next, we will quantize the background and obtain a quantum evolution equation for the composite state of the gravity-perturbation system. Finally, in the last step, we will extract an effective evolution equation for the field mode degrees of freedom, due to which we can extract an effective anisotropic background for propagation of the massive modes.

\subsection{Quantum Field on a Cosmological Classical Spacetime}

We start with considering a massive scalar field propagating on a more general classical, anisotropic background spacetime. In particular, we take a Bianchi type I model whose line element is represented by

$$
g_{a b} d x^{a} d x^{b}=-N_{x_{0}}^{2}\left(x_{0}\right)\left(d x_{0}\right)^{2}+\sum_{i}^{3} a_{i}^{2}\left(x_{0}\right)\left(d x^{i}\right)^{2},
$$

where $N_{x_{0}}$ is the lapse function and $a_{i}$ the scale factor in the $x^{i}$-direction. The coordinates $\left(x_{0}, \mathbf{x}\right)$ are chosen such that $x_{0} \in \mathbb{R}$ is a generic time coordinate, and $\mathbf{x} \in \mathbb{T}^{3}$ (i.e., a 3-torus with the coordinates $\left.x^{j} \in\left(0, \ell_{j}\right)\right)$.

We consider a real, minimally coupled scalar field, $\varphi\left(x_{0}, \mathbf{x}\right)$, with a mass $m$, propagating on the background (Equation(1)). Having the Lagrangian density for $\varphi\left(x_{0}, \mathbf{x}\right)$, with a quadratic potential,

$$
\mathcal{L}_{\varphi}=-\frac{1}{2}\left[g^{a b} \nabla_{a} \varphi \nabla_{b} \varphi+m^{2} \varphi^{2}\right],
$$


the equation of motion is obtained as

$$
\left(g^{a b} \nabla_{a} \nabla_{b}-m^{2}\right) \varphi=0 .
$$

On the $x_{0}=$ const slice, by performing the Legendre transformation, we take a canonically conjugate momentum $\pi_{\varphi}$ to the field $\varphi$. Then, the classical solutions of Equation (3) for the pair $\left(\varphi, \pi_{\varphi}\right)$ can be Fourier expanded as ${ }^{1}$

$$
\begin{aligned}
\varphi\left(x_{0}, \mathbf{x}\right) & =\frac{1}{\ell^{3 / 2}} \sum_{\mathbf{k} \in \mathscr{L}} \varphi_{\mathbf{k}}\left(x_{0}\right) e^{i \mathbf{k} \cdot \mathbf{x}}, \\
\pi_{\varphi}\left(x_{0}, \mathbf{x}\right) & =\frac{1}{\ell^{3 / 2}} \sum_{\mathbf{k} \in \mathscr{L}} \pi_{\mathbf{k}}\left(x_{0}\right) e^{i \mathbf{k} \cdot \mathbf{x}},
\end{aligned}
$$

with $\mathscr{L}=\mathscr{L}_{+} \cup \mathscr{L}_{-}$being a 3-dimensional lattice:

$$
\mathscr{L}_{+}=\left\{\mathbf{k}: k_{3}>0\right\} \cup\left\{\mathbf{k}: k_{3}=0, k_{2}>0\right\} \cup\left\{\mathbf{k}: k_{3}=k_{2}=0, k_{1}>0\right\},
$$

and

$$
\mathscr{L}_{-}=\left\{\mathbf{k}: k_{3}<0\right\} \cup\left\{\mathbf{k}: k_{3}=0, k_{2}<0\right\} \cup\left\{\mathbf{k}: k_{3}=k_{2}=0, k_{1}<0\right\},
$$

spanned by $\mathbf{k}=\left(k_{1}, k_{2}, k_{3}\right) \in(2 \pi \mathbb{Z} / \ell)^{3}$, where $\mathbb{Z}$ is the set of integers [13,18].

Let us decompose the field modes into the real and imaginary parts as

$$
\varphi_{\mathbf{k}}\left(x_{0}\right)=\frac{1}{\sqrt{2}}\left[\varphi_{\mathbf{k}}^{(1)}\left(x_{0}\right)+i \varphi_{\mathbf{k}}^{(2)}\left(x_{0}\right)\right]
$$

Then, the reality condition implies that $\varphi_{\mathbf{k}}^{(1)}=\varphi_{-\mathbf{k}}^{(1)}$ and $\varphi_{-\mathbf{k}}^{(2)}=-\varphi_{\mathbf{k}}^{(2)}$. By introducing a new variable $Q_{\mathbf{k}}$,

$$
Q_{\mathbf{k}}\left(x_{0}\right)= \begin{cases}\varphi_{\mathbf{k}}^{(1)}\left(x_{0}\right), & \text { if } \mathbf{k} \in \mathscr{L}_{+}, \\ \varphi_{-\mathbf{k}}^{(2)}\left(x_{0}\right), & \text { if } \mathbf{k} \in \mathscr{L}_{-}\end{cases}
$$

associated to the real variables $\varphi_{\mathbf{k}}^{(1)}$ and $\varphi_{\mathbf{k}}^{(2)}$, we can rewrite the field modes $\varphi_{\mathbf{k}}$ as

$$
\varphi_{\mathbf{k}}\left(x_{0}\right)=\frac{1}{\sqrt{2}}\left[Q_{\mathbf{k}}\left(x_{0}\right)+i Q_{-\mathbf{k}}\left(x_{0}\right)\right] .
$$

Likewise, the decomposition of the momentum $\pi_{\mathbf{k}}$ as

$$
\pi_{\mathbf{k}}\left(x_{0}\right)=\frac{1}{\sqrt{2}}\left[\pi_{\mathbf{k}}^{(1)}\left(x_{0}\right)+i \pi_{\mathbf{k}}^{(2)}\left(x_{0}\right)\right]
$$

in terms of the real variables $\pi_{\mathbf{k}}^{(1)}$ and $\pi_{\mathbf{k}}^{(2)}$, and introducing the new variable $P_{\mathbf{k}}$ (conjugate to $Q_{\mathbf{k}}$ above), as

$$
P_{\mathbf{k}}\left(x_{0}\right)= \begin{cases}\pi_{\mathbf{k}}^{(1)}\left(x_{0}\right), & \text { if } \mathbf{k} \in \mathscr{L}_{+} \\ \pi_{-\mathbf{k}}^{(2)}\left(x_{0}\right), & \text { if } \mathbf{k} \in \mathscr{L}_{-}\end{cases}
$$

yields

$$
\pi_{\mathbf{k}}\left(x_{0}\right)=\frac{1}{\sqrt{2}}\left[P_{\mathbf{k}}\left(x_{0}\right)+i P_{-\mathbf{k}}\left(x_{0}\right)\right]
$$

The conjugate variables $\left(Q_{\mathbf{k}}, P_{\mathbf{k}}\right)$ satisfy the Poisson bracket, $\left\{Q_{\mathbf{k}}, P_{\mathbf{k}^{\prime}}\right\}=\delta_{\mathbf{k}, \mathbf{k}^{\prime}}$. 
Using the Equations (7) and (10), the Hamiltonian of the scalar test field, $\varphi$, can be written as the sum of the Hamiltonians, $H_{\mathbf{k}}\left(x_{0}\right)$, of the decoupled harmonic oscillators, each given in terms of $\left(Q_{\mathbf{k}}, P_{\mathbf{k}}\right)$ :

$$
H_{\varphi}\left(x_{0}\right):=\sum_{\mathbf{k} \in \mathscr{L}} H_{\mathbf{k}}\left(x_{0}\right)=\frac{N_{x_{0}}\left(x_{0}\right)}{2\left|a_{1} a_{2} a_{3}\right|} \sum_{\mathbf{k} \in \mathscr{L}}\left(P_{\mathbf{k}}^{2}+\omega_{k}^{2}\left(x_{0}\right) Q_{\mathbf{k}}^{2}\right),
$$

where $\omega_{k}\left(x_{0}\right)$ is a time-dependent frequency, defined by

$$
\omega_{k}^{2}\left(x_{0}\right):=\left|a_{1} a_{2} a_{3}\right|^{2}\left[\sum_{i=1}^{3}\left(\frac{k_{i}}{a_{i}}\right)^{2}+m^{2}\right] .
$$

Note that $Q_{\mathbf{k}}$ is the field amplitude for the mode characterized by $\mathbf{k}$.

To quantize the field, we follow the Schrödinger representation. While the background spacetime is left as the classical, the quantization of $Q_{\mathbf{k}}$ for a fixed mode $\mathbf{k}$ resembles that of a quantum harmonic oscillator with the Hilbert space $\mathcal{H}_{\mathrm{Q}}^{(\mathbf{k})}=L^{2}\left(\mathbb{R}, d Q_{\mathbf{k}}\right)$, where $\left(Q_{\mathbf{k}}, P_{\mathbf{k}}\right)$ are promoted to operators on $\mathcal{H}_{Q}^{(\mathbf{k})}$ as

$$
\hat{Q}_{\mathbf{k}} \psi\left(Q_{\mathbf{k}}\right)=Q_{\mathbf{k}} \psi\left(Q_{\mathbf{k}}\right) \quad \text { and } \quad \hat{P}_{\mathbf{k}} \psi\left(Q_{\mathbf{k}}\right)=-i \hbar\left(\partial / \partial Q_{\mathbf{k}}\right) \psi\left(Q_{\mathbf{k}}\right)
$$

Then, the Hamiltonian operator $\hat{H}_{\mathbf{k}}$ generates the time evolution of the state $\psi\left(Q_{\mathbf{k}}\right)$ via the Schrödinger equation

$$
i \hbar \partial_{x_{0}} \psi\left(x_{0}, Q_{\mathbf{k}}\right)=\frac{\ell^{3} N_{x_{0}}}{2 V}\left(\hat{P}_{\mathbf{k}}^{2}+\omega_{k}^{2} \hat{Q}_{\mathbf{k}}^{2}\right) \psi\left(x_{0}, Q_{\mathbf{k}}\right),
$$

where $V=\ell^{3}\left|a_{1} a_{2} a_{3}\right|$ denotes the physical volume of the universe.

By setting $x_{0}=\phi$ in Equation (15) as an internal time parameter, the evolution of the state $\psi\left(Q_{\mathbf{k}}\right)$ with respect to $\phi$ on a Bianchi-I background with components $\left(\tilde{N}_{\phi}, \tilde{a}_{i}(\phi)\right)$ reads

$$
i \hbar \partial_{\phi} \psi\left(\phi, Q_{\mathbf{k}}\right)=\frac{\tilde{N}_{\phi}}{2\left|\tilde{a}_{1} \tilde{a}_{2} \tilde{a}_{3}\right|}\left[\hat{P}_{\mathbf{k}}^{2}+\tilde{\omega}_{k}^{2}(\phi) \hat{Q}_{\mathbf{k}}^{2}\right] \psi\left(\phi, Q_{\mathbf{k}}\right),
$$

where

$$
\tilde{\omega}_{k}^{2}(\phi)=\left(\sum_{i}^{3} \frac{\tilde{k}_{i}^{2}}{\tilde{a}_{i}^{2}}+\tilde{m}^{2}\right)\left(\tilde{a}_{1} \tilde{a}_{2} \tilde{a}_{3}\right)^{2} .
$$

Clearly, one gets an isotropic background for the field by $\tilde{a}_{1}(\phi)=\tilde{a}_{2}(\phi)=\tilde{a}_{3}(\phi) \equiv \tilde{a}(\phi)$.

\subsection{Quantization of the Background}

In our model herein this paper, we will assume that the field, $\varphi$, propagates on an isotropic FLRW spacetime in a super-Planckian regime so that this isotropic background has to be quantized. However, the reason for constructing a general formalism of the field on an anisotropic background in the previous subsection is for the purpose of comparison, when an effectively dressed spacetime emerges from the isotropic quantum background. We will show that the emergent effective spacetime can have the same structure of an anisotropic Bianchi-I geometry for the field propagation.

Let us assume a harmonic time gauge, $x_{0}=\tau$, and set the isotropic components as $a_{1}=a_{2}=a_{3}=a(\tau)$ in Equation (1). Then, $N_{\tau}=a^{3}(\tau)$, and the Hamiltonian (12) becomes

$$
H_{\varphi}^{(\text {iso })}=\sum_{\mathbf{k}} H_{\tau, \mathbf{k}}:=\frac{1}{2} \sum_{\mathbf{k}}\left(P_{\mathbf{k}}^{2}+\omega_{\tau, k}^{2} Q_{\mathbf{k}}^{2}\right)
$$

where

$$
\omega_{\tau, k}^{2}(\tau)=k^{2} a^{4}(\tau)+m^{2} a^{6}(\tau) .
$$


We note that the massless scalar field, $\phi(\tau)$, still serves as an internal time parameter.

In quantum theory, we will quantize not only the test field but also the background geometry. We will assume that the backreaction of the quantum field on the background quantized spacetime is negligible. This yields an evolution for the wave function, $\psi\left(Q_{\mathbf{k}}\right)$, of the test field with respect to the internal time $\phi$. Let $\mathcal{H}_{\text {kin }}^{o}=\mathcal{H}_{\text {grav }} \otimes \mathcal{H}_{\phi}$ denote the background Hilbert space, which consists of the Hilbert space of the gravity sector and that of the scalar clock variable $\phi$; the matter sector is quantized according to the Schrödinger representation, $\mathcal{H}_{\phi}=L^{2}(\mathbb{R}, d \phi)$. Likewise, the Hilbert space of the field modes reads $\mathcal{H}_{\varphi}^{(\mathbf{k})}=L^{2}\left(\mathbb{R}, d Q_{\mathbf{k}}\right)$, as before. Subsequently, the full kinematical Hilbert space of the system for a single mode $\mathbf{k}$ is given by $\mathcal{H}_{\text {kin }}^{(\mathbf{k})}=\mathcal{H}_{\text {kin }}^{o} \otimes \mathcal{H}_{\varphi}^{(\mathbf{k})}$.

In LQC, the background Hamiltonian constraint operator,

$$
\hat{\mathcal{C}}_{o}=\hat{\mathcal{C}}_{\text {grav }}+\hat{\mathcal{C}}_{\phi}
$$

is well-defined on $\mathcal{H}_{\mathrm{kin}}^{o}$; the physical states $\Psi_{o}(\phi, v) \in \mathcal{H}_{\mathrm{kin}}^{o}$ are those lying on the kernel of $\hat{\mathcal{C}}_{o}$ and are solutions to the self-adjoint constraint Equation: [30]

$$
N_{\tau} \hat{\mathcal{C}}_{o} \Psi_{o}(v, \phi)=-\frac{\hbar^{2}}{2 \ell^{3}}\left(\partial_{\phi}^{2}+\Theta\right) \Psi_{o}(v, \phi)=0 .
$$

The quantum number $v$ is the eigenvalue of the background volume operator, $\hat{v}_{o}=\widehat{\ell^{3} a^{3}}$, which acts on the states $\Psi_{o}(\phi, v) \in \mathcal{H}_{\text {kin }}^{o}$ as

$$
\hat{V}_{o} \Psi_{o}(\nu, \phi)=2 \pi \gamma \ell_{\mathrm{Pl}}|v| \Psi_{o}(\nu, \phi) .
$$

Moreover, $\Theta$ is a difference operator that acts on $\Psi_{o}(v)$, involving only the volume sector $v$. Taking only the positive frequency solutions to Equation (21), we get a Schrödinger equation for the background as

$$
-i \hbar \partial_{\phi} \Psi_{o}(v, \phi)=\hbar \sqrt{\Theta} \Psi_{o}(v, \phi)=: \hat{H}_{o} \Psi_{o}(v, \phi) .
$$

The solutions yield a physical Hilbert space, $\mathcal{H}_{\text {phys }}^{o}$ equipped by the inner product

$$
\left\langle\Psi_{o} \mid \Psi_{o}^{\prime}\right\rangle=\sum_{v} \Psi_{o}^{*}\left(v, \phi_{0}\right) \Psi_{o}^{\prime}\left(v, \phi_{0}\right),
$$

for an "instant" $\phi_{0}$ of the internal time.

For a composite state $\Psi\left(v, Q_{\mathbf{k}}, \phi\right) \in \mathcal{H}_{\text {kin }}^{(\mathbf{k})}$ of the geometry-test field system, the action of the total quantum Hamiltonian constraint, $\hat{\mathcal{C}}_{\tau, \mathbf{k}}$, is written as [13]

$$
\hat{\mathcal{C}}_{\tau, \mathbf{k}} \Psi\left(\nu, Q_{\mathbf{k}}, \phi\right)=\left(N_{\tau} \hat{\mathcal{C}}_{o}+\hat{H}_{\tau, \mathbf{k}}\right) \Psi\left(\nu, Q_{\mathbf{k}}, \phi\right)=0,
$$

where $\hat{H}_{\tau, \mathbf{k}}$ is the Hamiltonian operator of the kth field mode,

$$
\hat{H}_{\tau, \mathbf{k}}=\frac{1}{2}\left[\hat{P}_{\mathbf{k}}^{2}+\left(k^{2} \hat{a}^{4}+m^{2} \hat{a}^{6}\right) \hat{Q}_{\mathbf{k}}^{2}\right] .
$$

By replacing Equation (21) into the constraint Equation (25), we obtain

$$
-i \hbar \partial_{\phi} \Psi\left(v, Q_{\mathbf{k}}, \phi\right)=\left[\hat{H}_{o}^{2}-2 \ell^{3} \hat{H}_{\tau, \mathbf{k}}\right]^{\frac{1}{2}} \Psi\left(v, Q_{\mathbf{k}}, \phi\right),
$$

which represents a quantum evolution of $\Psi\left(v, Q_{\mathbf{k}}, \phi\right)$ with respect to the internal time $\phi$. In a test field approximation, when the backreaction effect is omitted, the expression under the square root can be expanded up to the first-order terms, as [13]

$$
-i \hbar \partial_{\phi} \Psi\left(\nu, Q_{\mathbf{k}}, \phi\right) \approx\left(\hat{H}_{o}-\hat{H}_{\phi, \mathbf{k}}\right) \Psi\left(\nu, Q_{\mathbf{k}}, \phi\right) .
$$


In expanding the right-hand-side of Equation (27) to derive the equation above, we regarded $\hat{H}_{0}$ as the Hamiltonian of the heavy degree of freedom, whereas $\hat{H}_{\phi, \mathbf{k}}$, defined by

$$
\hat{H}_{\phi, \mathbf{k}}:=\ell^{3} \hat{H}_{o}^{-\frac{1}{2}} \hat{H}_{\tau, \mathbf{k}} \hat{H}_{o}^{-\frac{1}{2}},
$$

was considered as the Hamiltonian of the light degree of freedom (i.e, a perturbation term). In this approximation, it is suitable to separate the total state of the system as

$$
\Psi\left(v, Q_{\mathbf{k}}, \phi\right)=\Psi_{o}(v, \phi) \otimes \psi\left(Q_{\mathbf{k}}, \phi\right) .
$$

To explore the quantum evolution of a pure test field state, $\psi\left(Q_{\mathbf{k}}, \phi\right)$, on a timedependent background, it is more convenient to employ an interaction picture. Thus, we introduce

$$
\Psi_{\text {int }}\left(\nu, Q_{\mathbf{k}}, \phi\right)=e^{-\left(i \hat{H}_{o} / \hbar\right)\left(\phi-\phi_{0}\right)} \Psi\left(\nu, Q_{\mathbf{k}}, \phi\right),
$$

In this picture, the geometry evolves by $\hat{H}_{0}$ through Equation (23) for any $\Psi_{o} \in \mathcal{H}_{\text {kin }}^{o}$ in the Heisenberg picture,

$$
\Psi_{o}(\nu, \phi)=e^{\left(i \hat{H}_{o} / \hbar\right)\left(\phi-\phi_{0}\right)} \Psi_{o}\left(\nu, \phi_{0}\right) .
$$

Plugging this into Equation (31), we get

$$
\Psi_{\text {int }}\left(\nu, Q_{\mathbf{k}}, \phi\right)=\Psi_{o}\left(\nu, \phi_{0}\right) \otimes \psi\left(Q_{\mathbf{k}}, \phi\right) .
$$

Thereby, the geometrical sector in the composite state $\Psi_{\text {int }}\left(\nu, Q_{\mathbf{k}}, \phi\right)$ becomes frozen in the instant of time $\phi_{0}$ so that $\Psi_{\text {int }}$ represents a time-dependent test field state, $\psi$, solely.

By replacing Equation (33) into the evolution Equation (28) and tracing out the geometrical state, $\Psi_{o}\left(v, \phi_{0}\right)$, a quantum evolution for $\psi\left(Q_{\mathbf{k}}, \phi\right)$ is obtained as

$$
i \hbar \partial_{\phi} \psi=\frac{1}{2}\left[\left\langle\hat{H}_{o}^{-1}\right\rangle \hat{P}_{\mathbf{k}}^{2}+\left(k^{2}\left\langle\hat{H}_{o}^{-\frac{1}{2}} \hat{a}^{4}(\phi) \hat{H}_{o}^{-\frac{1}{2}}\right\rangle+m^{2}\left\langle\hat{H}_{o}^{-\frac{1}{2}} \hat{a}^{6}(\phi) \hat{H}_{o}^{-\frac{1}{2}}\right\rangle\right) \hat{Q}_{\mathbf{k}}^{2}\right] \psi,
$$

where $\langle\cdot\rangle$ denotes the expectation value with respect to $\Psi_{o}\left(v, \phi_{0}\right)$. It is clear that the use of the interaction picture in Equation (34) provided the Heisenberg description for the quantum geometrical elements; that is, the geometry state, $\Psi_{o}\left(\nu, \phi_{0}\right)$, is fixed at time $\phi=\phi_{0}$, while the geometrical operator, $\hat{a}(\phi)=\hat{V}_{o}^{1 / 3}(\phi) / \ell$, evolves in time as

$$
\hat{a}(\phi)=e^{-\left(i \hat{H}_{o} / \hbar\right)\left(\phi-\phi_{0}\right)} \hat{a} e^{\left(i \hat{H}_{o} / \hbar\right)\left(\phi-\phi_{0}\right)} .
$$

Therefore, Equation (34) represents a $\phi$-evolution of the field, $\psi\left(Q_{\mathbf{k}}, \phi\right)$, on a timedependent (classical) background, which is similar to the one we had in classical spacetime (cf. Equation (16)).

\subsection{Emergence of Anisotropic Dressed Spacetimes}

Equation (34) can be interpreted as an evolution equation for the field modes on an (effective) classical spacetime, whose components are generated by the expectation values of the original isotropic quantum geometry operators with respect to the unperturbed state $\Psi_{0}$. To explore the properties of such effective spacetime, we can compare Equation (34), for the evolution of the state $\psi$, with the corresponding Equation (16), for the same state $\psi$, on an anisotropic classical background. This comparison yields a set of relations between parameters of the Bianchi I geometry, $\left(\tilde{N}_{\phi}, \tilde{a}_{i}, \tilde{k}_{i}, \tilde{m}\right)$, and those of the isotropic quantum geometry, $\left(\hat{a}, \hat{H}_{0}, k, m\right)$, as 


$$
\begin{aligned}
\tilde{N}_{\phi} & =\ell^{3}\left|\tilde{a}_{1} \tilde{a}_{2} \tilde{a}_{3}\right|\left\langle\hat{H}_{o}^{-1}\right\rangle, \\
\sum_{i=1}^{3} \frac{\tilde{k}_{i}^{2}}{\tilde{a}_{i}^{2}} \tilde{N}_{\phi}\left|\tilde{a}_{1} \tilde{a}_{2} \tilde{a}_{3}\right| & =\sum_{i=1}^{3} k_{i}^{2} \ell^{3}\left\langle\hat{H}_{o}^{-1 / 2} \hat{a}^{4} \hat{H}_{o}^{-1 / 2}\right\rangle, \\
\tilde{N}_{\phi} \tilde{m}^{2}\left|\tilde{a}_{1} \tilde{a}_{2} \tilde{a}_{3}\right| & =\ell^{3} m^{2}\left\langle\hat{H}_{o}^{-1 / 2} \hat{a}^{6} \hat{H}_{o}^{-1 / 2}\right\rangle .
\end{aligned}
$$

Note that $\hat{a} \equiv \hat{a}(\phi)$ and $\left\langle\hat{H}_{o}^{-1}\right\rangle=\left(\tilde{p}_{\phi}\right)^{-1}$. Equation (36a-c) provide an underdetermined system of five equations with eight unknowns $\left(\tilde{N}_{\phi}, \tilde{a}_{i}, \tilde{k}_{i}, \tilde{m}\right)$. Thus, to be able to solve this system, we need to impose some arbitrary conditions on these parameters to reduce the number of unknowns to five. Different classes of solutions by imposing various conditions on the variables and their physical consequences were discussed in [24]. As an example, we will present two classes of such solutions; one is produced by a massless test field, $m=\tilde{m}=0$, and the other is provided by the dressed massive modes, $\tilde{m}, m \neq 0$.

For a massless test field, $m=0$, we will immediately obtain $\tilde{m}=0$. In this case, we will have four equations for the seven unknown parameters $\left(\tilde{N}_{\phi}, \tilde{a}_{i}, \tilde{k}_{i}\right)$. Therefore, we will still need three more conditions to be able to solve the system in Equation $(36 \mathrm{a}, \mathrm{b})$. The simplest choice is $k_{i}^{2}=\tilde{k}_{i}^{2}$ (for each $i$ ) so that $\tilde{a}_{1}^{2}=\tilde{a}_{2}^{2}=\tilde{a}_{3}^{2}=\tilde{a}^{2}$. Then, we obtain

$$
\begin{aligned}
\tilde{a}^{4} & =\frac{\left\langle\hat{H}_{o}^{-1 / 2} \hat{a}^{4}(\phi) \hat{H}_{o}^{-1 / 2}\right\rangle}{\left\langle\hat{H}_{o}^{-1}\right\rangle}, \\
\tilde{N}_{\phi} & =\ell^{3}\left\langle\hat{H}_{o}^{-1}\right\rangle^{\frac{1}{4}}\left\langle\hat{H}_{o}^{-1 / 2} \hat{a}^{4}(\phi) \hat{H}_{o}^{-1 / 2}\right\rangle^{\frac{3}{4}}=\ell^{3} \tilde{p}_{\phi}^{-1} \tilde{a}^{3} \equiv \bar{N}_{\phi} .
\end{aligned}
$$

If $m, \tilde{m} \neq 0$ and $\tilde{m} \neq m$, we will have different ranges of solutions (cf. [24]). However, for our purpose in this paper, we will consider only a specific solution by imposing the condition $\tilde{k}_{i}=\alpha_{i i} k_{i}$ (with $i=1,2,3$ ). This yields

$$
\begin{aligned}
\tilde{N}_{\phi} & =\frac{\bar{N}_{\phi}}{\lambda}, \\
\tilde{a}_{i} & =\frac{\alpha_{i i}}{\lambda} \tilde{a}, \\
\tilde{m}^{4} & =m^{4} \lambda^{4} \frac{\left\langle\hat{H}_{o}^{-1 / 2} \hat{a}^{6}(\phi) \hat{H}_{o}^{-1 / 2}\right\rangle^{2}\left\langle\hat{H}_{o}^{-1}\right\rangle}{\left\langle\hat{H}_{o}^{-1 / 2} \hat{a}^{4}(\phi) \hat{H}_{o}^{-1 / 2}\right\rangle^{3}},
\end{aligned}
$$

where $\lambda \equiv\left(\alpha_{11} \alpha_{22} \alpha_{33}\right)^{1 / 2}$ is a constant. Note that, as a special subcase, when $\alpha_{i i}=1$ (so $\lambda=1)$, the wave vector becomes undressed, $\tilde{k}_{i}=k_{i}$, and an isotropic dressed scale factor, $\tilde{a}_{1}=\tilde{a}_{2}=\tilde{a}_{3}=\tilde{a}$, identical to Equation (37a), is obtained. However, here, different from the isotropic case above for $m=0$, a nonzero dressed mass is obtained as

$$
\bar{m}^{2}=m^{2} \frac{\left\langle\hat{H}_{o}^{-1 / 2} \hat{a}^{6}(\phi) \hat{H}_{o}^{-1 / 2}\right\rangle\left\langle\hat{H}_{o}^{-1}\right\rangle^{\frac{1}{2}}}{\left\langle\hat{H}_{o}^{-1 / 2} \hat{a}^{4}(\phi) \hat{H}_{o}^{-1 / 2}\right\rangle^{\frac{3}{2}}}=\frac{\tilde{m}^{2}}{\lambda^{2}} .
$$

This solution represents an isotropic dressed spacetime with the scale factor $\bar{a}(\phi)$, over which a massive mode with the mass $\bar{m}$ and an undressed wave-vector $\mathbf{k}=\left(k_{1}, k_{2}, k_{3}\right)$ propagate.

\section{QFT on the Dressed Spacetime}

To date, we have seen that the massive field modes propagating on an isotropic quantum geometry can explore a classical anisotropic dressed background, $\tilde{g}_{a b}$ (see, e.g., Equation $(38 \mathrm{a}-\mathrm{c})$, which is the solution of Equation $(36 \mathrm{a}-\mathrm{c})$. In this section, we will study the QFT and the issue of the gravitational particle productions on such an emergent anisotropic spacetime. 


\subsection{Field Equation on the Dressed Anisotropic Background}

We suppose that the scalar field, $\varphi$, propagates on the dressed Bianchi I background

$$
\tilde{g}_{a b} d x^{a} d x^{b}=-\tilde{N}_{\phi}^{2}(\phi) d \phi^{2}+\sum_{i=1}^{3} \tilde{a}_{i}^{2}(\phi)\left(d x^{i}\right)^{2},
$$

whose components are given as the solutions to Equation (36). For convenience, we introduce the new variables

$$
\tilde{c}(\phi):=\left(\tilde{a}_{1} \tilde{a}_{2} \tilde{a}_{3}\right)^{\frac{2}{3}}=\left(\tilde{c}_{1} \tilde{c}_{2} \tilde{c}_{3}\right)^{\frac{1}{3}} \text { and } \quad \tilde{c}_{i}(\phi):=\tilde{a}_{i}^{2}(\phi),
$$

and consider a conformal time parameter, $\tilde{\eta}$, defined by

$$
\tilde{N}_{\phi} d \phi=\tilde{N}_{\tilde{\eta}} d \tilde{\eta}=\tilde{c}^{1 / 2} d \tilde{\eta} \quad \Rightarrow \quad d \tilde{\eta}=\ell^{3}\left\langle\hat{H}_{o}^{-1}\right\rangle \tilde{c}(\phi) d \phi .
$$

Let us now take an auxiliary field, $\chi_{\mathbf{k}} \equiv \sqrt{\tilde{c}(\tilde{\eta})} \varphi_{\mathbf{k}}$. Then, in terms of the above variables, the equation of motion reads

$$
\chi_{\mathbf{k}}^{\prime \prime}+\left[\tilde{\omega}_{\tilde{\eta}, k}^{2}(\tilde{\eta})-\frac{\tilde{c}^{\prime \prime}}{2 \tilde{c}}+\frac{\tilde{c}^{2}}{4 \tilde{c}^{2}}\right] \chi_{\mathbf{k}}=0,
$$

where a prime stands for a differentiation with respect to $\tilde{\eta}$, and $\tilde{\omega}_{\tilde{\eta}, k}$ is given by

$$
\tilde{\omega}_{\tilde{\eta}, k}^{2}(\tilde{\eta})=\tilde{c}^{-2}\left[k^{2} \frac{\left\langle\hat{H}_{o}^{-\frac{1}{2}} \hat{a}^{4} \hat{H}_{o}^{-\frac{1}{2}}\right\rangle}{\left\langle\hat{H}_{o}^{-1}\right\rangle}+m^{2} \frac{\left\langle\hat{H}_{o}^{-\frac{1}{2}} \hat{a}^{6} \hat{H}_{o}^{-\frac{1}{2}}\right\rangle}{\left\langle\hat{H}_{o}^{-1}\right\rangle}\right] .
$$

Notice that in simplifying equations above, we have used the relations between components according to Equation (36).

The frequency from Equation (44) is specified only when the solutions for $\tilde{c}(\tilde{\eta})$ are determined. It turns out that only two classes of solutions for $\tilde{c}$ exist, which depend on the conditions on the field mass:

(i) For massive field with undressed mass, $\tilde{m}=m \neq 0$, the relation for $\tilde{c}(\tilde{\eta})$ has the form

$$
\tilde{c}(\tilde{\eta})=\frac{\left\langle\hat{H}_{o}^{-\frac{1}{2}} \hat{a}^{6} \hat{H}_{o}^{-\frac{1}{2}}\right\rangle^{\frac{1}{3}}}{\left\langle\hat{H}_{o}^{-1}\right\rangle^{\frac{1}{3}}} .
$$

(ii) For a massive field with the dressed mass, $\tilde{m} \neq m$, or a massless field, $\tilde{c}(\tilde{\eta})$ has the solutions of the form

$$
\tilde{c}(\tilde{\eta})=\xi^{2} \frac{\left\langle\hat{H}_{o}^{-\frac{1}{2}} \hat{a}^{4} \hat{H}_{o}^{-\frac{1}{2}}\right\rangle^{\frac{1}{2}}}{\left\langle\hat{H}_{o}^{-1}\right\rangle^{\frac{1}{2}}}=\xi^{2} \tilde{a}^{2}(\tilde{\eta}),
$$

where $\xi$ is a parameter that distinguishes the anisotropic solutions from the isotropic one and depends on the conditions imposed on the additional unknown variables $\tilde{a}_{i}{ }^{\prime}$ s and $\tilde{k}_{i}$ 's of the system in Equation (36). The case $\tilde{\xi}^{2}=1$ associates with the isotropic solution, whereas the case $\xi \neq 1$ denotes the anisotropic solutions (cf. Equations (37) and (39) for different classes of the solutions).

\subsection{The Adiabatic Condition and the Vacuum State}

Any solution $\chi_{\mathbf{k}}(\tilde{\eta})$ to the Klein-Gordon equation (Equation (43)) can be expanded as

$$
\chi_{\mathbf{k}}(\tilde{\eta})=\frac{1}{\sqrt{2}}\left[a_{\mathbf{k}} u_{k}^{*}(\tilde{\eta})+a_{-\mathbf{k}}^{*} u_{k}(\tilde{\eta})\right],
$$


where $a_{\mathbf{k}}$ and $a_{\mathbf{k}}^{*}$ are constants of integration, and $u_{k}(\tilde{\eta})$ are the general mode solutions of Equation (43),

$$
u_{k}^{\prime \prime}+\left(\tilde{\omega}_{\tilde{\eta}, k}^{2}(\tilde{\eta})-\mathcal{Q}(\tilde{\eta})\right) u_{k}=0,
$$

with $\mathcal{Q} \equiv \tilde{c}^{\prime \prime} / 2 \tilde{c}-\tilde{c}^{2} / 4 \tilde{c}^{2}$. These modes, together with their complex conjugates, $u_{k}^{*}(\tilde{\eta})$, form a complete set of orthonormal basis under the scalar product

$$
W\left(u_{k}^{*}, u_{k}\right):=u_{k} u_{k}^{* \prime}-u_{k}^{*} u_{k}^{\prime}=2 i .
$$

In quantum theory, $a_{\mathbf{k}}$ and $a_{\mathbf{k}}^{*}$ become annihilation and creation operators, $\hat{a}_{\mathbf{k}}$ and $\hat{a}_{\mathbf{k}^{\prime}}^{+}$ satisfying the commutation relation

$$
\left[\hat{a}_{\mathbf{k}}, \hat{a}_{\mathbf{k}^{\prime}}^{\dagger}\right]=\hbar \ell^{3} \delta_{\mathbf{k}, \mathbf{k}^{\prime}}
$$

where all other commutation relations vanish. For the positive frequency solutions, a choice of the basis, $u_{k}(\tilde{\eta})$, determines a vacuum state, $|0\rangle$, which is defined as the eigenstate of the annihilation operators obeying the equation $\hat{a}_{\mathbf{k}}|0\rangle=0$. Different families of the mode solutions distinguish different vacuum states. Accordingly, a Fock space is generated by repeatedly acting the operator $\hat{a}_{\mathbf{k}}^{+}$on a chosen vacuum state. Thereby, the evolution of the quantum fields on the quantum-gravity-induced dressed spacetime is determined. Now, the general (auxiliary) field solutions, $\chi(\tilde{\eta}, \mathbf{x})$, can be expanded as

$$
\chi(\tilde{\eta}, \mathbf{x})=\frac{1}{\ell^{3}} \sum_{\mathbf{k}} \chi_{\mathbf{k}}(\tilde{\eta}, \mathbf{x}),
$$

where

$$
\chi_{\mathbf{k}}=\frac{1}{\sqrt{2}}\left[a_{\mathbf{k}} u_{k}^{*}(\tilde{\eta}) e^{i \mathbf{k} \cdot \mathbf{x}}+a_{\mathbf{k}}^{*} u_{k}(\tilde{\eta}) e^{-i \mathbf{k} \cdot \mathbf{x}}\right] .
$$

To have a well-defined vacuum state for the mode solutions, the (effective) frequency

$$
\Omega_{k}^{2}(\tilde{\eta})=\tilde{\omega}_{\tilde{\eta}, k}^{2}(\tilde{\eta})-\mathcal{Q}(\tilde{\eta})
$$

should be positive. Thus, the wave-number must satisfy $k^{2} \geq k_{*}^{2}$, where

$$
k_{*}^{2}(\tilde{\eta}):=\frac{\mathcal{Q}(\tilde{\eta}) \tilde{c}^{2}(\tilde{\eta})\left\langle\hat{H}_{o}^{-1}\right\rangle}{\left\langle\hat{H}_{o}^{-\frac{1}{2}} \hat{a}^{4} \hat{H}_{o}^{-\frac{1}{2}}\right\rangle}-m^{2} \frac{\left\langle\hat{H}_{o}^{-\frac{1}{2}} \hat{a}^{6} \hat{H}_{o}^{-\frac{1}{2}}\right\rangle}{\left\langle\hat{H}_{o}^{-\frac{1}{2}} \hat{a}^{4} \hat{H}_{o}^{-\frac{1}{2}}\right\rangle},
$$

introduces a physical wavelength, $\lambda_{*}(\tilde{\eta}) \equiv 2 \pi \sqrt{\tilde{c}(\tilde{\eta})} / k_{*}$, which provides an upper bound for the wavelengths of the physical mode functions, i.e., $\lambda \leq \lambda_{*}$. Therefore, modes with short wavelengths (i.e., large momenta), $\lambda \ll \lambda_{*}$, characterize the vacuum states in short distances, i.e., the ultra-violet (UV) regimes. This regime contains a limit of arbitrary slow time, $\tilde{\eta}$, variation of the metric functions called the adiabatic regime. We will, henceforth, explore the mode functions describing the physical vacuum and particles associated with this adiabatic regime.

A positive-frequency, adiabatic vacuum mode can be defined by a generalized WKB approximate solution to the Klein-Gordon equation, as in [31],

$$
\underline{u}_{k}(\tilde{\eta})=\frac{1}{\sqrt{W_{k}(\tilde{\eta})}} \exp \left(-i \int^{\tilde{\eta}} W_{k}(\eta) d \eta\right) .
$$

Substituting this relation in Equation (48), we obtain a relation for $W_{k}(\eta)$ as

$$
\frac{W_{k}^{\prime \prime}}{W_{k}}-\frac{W_{k}^{\prime}}{W_{k}^{2}}-\frac{1}{2} \frac{W_{k}^{\prime 2}}{W_{k}^{2}}+2\left(W_{k}^{2}-\Omega_{k}^{2}\right)=0
$$


An appropriate function can be chosen for $W_{k}(\tilde{\eta})$, such as [32]

$$
W_{k}(\tilde{\eta})=\left[Y\left(1+\underline{\epsilon_{2}}\right)\left(1+\underline{\epsilon_{4}}\right)\right]^{\frac{1}{2}},
$$

where $Y \equiv \Omega_{k}^{2}=\tilde{\omega}_{\tilde{\eta}, k}^{2}-\mathcal{Q}$ and

$$
\begin{aligned}
& \underline{\epsilon_{2}}:=-Y^{-\frac{3}{4}} \partial_{\tilde{\eta}}\left(Y^{-\frac{1}{2}} \partial_{\tilde{\eta}} Y^{\frac{1}{4}}\right), \\
& \underline{\epsilon_{4}}:=-Y^{-\frac{1}{2}}\left(1+\underline{\epsilon_{2}}\right)^{-\frac{3}{4}} \partial_{\tilde{\eta}}\left\{\left[Y\left(1+\underline{\epsilon_{2}}\right)\right]^{-\frac{1}{2}} \partial_{\tilde{\eta}}\left(1+\underline{\epsilon_{2}}\right)^{\frac{1}{4}}\right\} .
\end{aligned}
$$

To get the solutions for $\underline{u}_{k}(\tilde{\eta})$, one needs to solve Equation (56) for $W(\tilde{\eta})$. However, instead of solving (56) exactly, one way is to generate asymptotic series in orders of time $\tilde{\eta}$ derivatives of the background dressed metric. Terminating this series at a given order will specify an adiabatic mode $\underline{u}_{k}(\tilde{\eta})$ to that order. Up to an order $n$ (being the power of $\tilde{\eta}$ derivatives of $\tilde{a}_{i}$ ), denoted $\mathcal{O}\left(\sqrt{\tilde{c}} / k \lambda_{n+\varepsilon}\right)^{n+\varepsilon}$ (with positive real number $\varepsilon$ ), the asymptotic adiabatic expansion of $W_{k}$ is defined to match

$$
W_{k}(\tilde{\eta})=W_{k}^{(0)}+W_{k}^{(2)}+\cdots+W_{k}^{(n)},
$$

and is obtained for higher order estimates by iteration.

When computing the expectation value of the energy-momentum operator of the scalar field, $\left\langle\tilde{0}\left|\hat{T}_{a b}\right| \tilde{0}\right\rangle$, with respect to the adiabatic vacuum, $|\tilde{0}\rangle$, associated to the mode function (Equation (55)), one encounters the UV divergences issues. All of these UV divergences are contained within the terms of adiabatic order equal to and smaller than four (cf. [33] and the appendix of Ref. [24]). Thus, the (adiabatic) regularization of the energy density and pressure of the scalar field are obtained from subtractions of the divergences contained within the adiabatic terms up to the fourth order; for example, the renormalized energy density of the field is given by

$$
\left\langle\tilde{0}\left|\hat{\rho}_{\varphi}\right| \tilde{0}\right\rangle_{\mathrm{ren}}=\frac{\hbar}{\ell^{3} \tilde{c}^{2}} \sum_{k}\left(\rho_{k}\left[u_{k}(\tilde{\eta})\right]-\underline{\rho_{k}}\left[\underline{u_{k}}(\tilde{\eta})\right]\right),
$$

where $\rho_{k}$ is the energy density of each mode, $u_{k}(\tilde{\eta})$, and $\rho_{k}$ is the energy density associated with the mode, $u_{k}$, up to the fourth adiabatic order. We will, thus, restrict ourselves to the fourth-order adiabatic states only ${ }^{2}$. Then, by setting $n=4$, we define $W_{k}(\tilde{\eta})$ to match the terms in Equation (60) that fall slowly in $\tilde{\omega}_{\tilde{\eta}, k}$, rather than considering the exact mode solutions of Equation (48). Accordingly, we consider an appropriate asymptotic condition (up to the order four) at which the field's exact mode functions, $u_{k}(\tilde{\eta})$, match the adiabatic functions, $\underline{u}_{k}(\tilde{\eta})$, as

$$
\begin{aligned}
& \left|u_{k}\left(\tilde{\eta}_{b}\right)\right|=\left|\underline{u}_{k}\left(\tilde{\eta}_{b}\right)\right|\left(1+\mathcal{O}\left(\sqrt{\tilde{c}} / k \lambda_{4+\varepsilon}\right)^{4+\varepsilon}\right), \\
& \left|u_{k}^{\prime}\left(\tilde{\eta}_{b}\right)\right|=\left|\underline{u}_{k}^{\prime}\left(\tilde{\eta}_{b}\right)\right|\left(1+\mathcal{O}\left(\sqrt{\tilde{c}} / k \lambda_{4+\varepsilon}\right)^{4+\varepsilon}\right),
\end{aligned}
$$

where $\tilde{\eta}=\tilde{\eta}_{b}$ is a natural choice for the preferred instant of time given at the quantum bounce in LQC. When a mode function, $u_{k}(\tilde{\eta})$, satisfies the conditions (62) at some initial time $\tilde{\eta}_{b}$, it will satisfy it for all times $\tilde{\eta}$. Therefore, an observable vacuum, $|0\rangle$, associated to $u_{k}(\tilde{\eta})$ will be of the fourth order for all times.

Following the above discussion, we thus discard the adiabatic order terms higher than four in Equation (57) and write

$$
W_{k}(\tilde{\eta})=\tilde{\omega}_{\tilde{\eta}, k}\left(1+\epsilon_{2}+\epsilon_{4}\right)^{\frac{1}{2}},
$$


where $\epsilon_{2}, \epsilon_{4}$ are defined by

$$
\begin{aligned}
\epsilon_{2} & =\underline{\epsilon_{2}}-\tilde{\omega}_{\tilde{\eta}, k}^{-2} \mathcal{Q} \\
& =-\frac{1}{4} Y^{-2} Y^{\prime \prime}+\frac{5}{16} Y^{-3}\left(Y^{\prime}\right)^{2}-\tilde{\omega}_{\tilde{\eta}, k}^{-2} \mathcal{Q}, \\
\epsilon_{4} & =\underline{\epsilon_{4}}-\underline{\epsilon_{2}} \tilde{\omega}_{\tilde{\eta}, k}^{-2} \mathcal{Q} \\
& =-\frac{1}{4} Y^{-1}\left(1+\underline{\epsilon_{2}}\right)^{-2}\left[\underline{\epsilon}^{\prime \prime}-\frac{1}{2} Y^{-1} Y^{\prime} \underline{\epsilon}^{\prime}-\frac{5}{4}\left(1+\underline{\epsilon_{2}}\right)^{-1}\left(\underline{\epsilon_{2}}\right)^{2}\right]-\underline{\epsilon_{2}} \tilde{\omega}_{\tilde{\eta}, k}^{-2} \mathcal{Q},
\end{aligned}
$$

and $\underline{\epsilon_{2}}$ is given by

$$
\underline{\epsilon_{2}}=-\frac{1}{4} Y^{-2} Y^{\prime \prime}+\frac{5}{16} Y^{-3}\left(Y^{\prime}\right)^{2}
$$

The leading order term of $\epsilon_{2}$ is of the second order, whereas the leading order in $\underline{\epsilon_{4}}$ is four. Thus, $\epsilon_{2}$ contains terms of orders two, four and higher, i.e,

$$
\epsilon_{2}=\epsilon_{2}^{(2)}+\epsilon_{2}^{(4)}+\text { Higher order terms, }
$$

and $\epsilon_{4}$ contains the leading order term four, i.e.,

$$
\epsilon_{4}=\epsilon_{4}^{(4)}+\text { Higher order terms. }
$$

It should be noticed that we have considered only terms until the fourth order in their expressions (for more details, see [24]).

\section{Gravitational Particle Production}

Our aim in this section is to study the gravitational particle production in the herein quantum gravity regime due to quantum field on the anisotropic dressed background.

Once a vacuum state, $|\tilde{0}\rangle$, is specified due to the positive frequency solutions, $\underline{v}_{k}(\tilde{\eta})$, of Equation (48), a Fock space, $\mathcal{H}_{\mathrm{F}}$, for the quantum field $\varphi$ is generated. Let $\left\{v_{k}\right\}$ and $\left\{\underline{v}_{k}\right\}$ be two sets of WKB solutions given by Equation (55) in the herein adiabatic regime. These mode functions form two normalized bases ${ }^{3}$ for $\mathcal{H}_{\mathrm{F}}$, so they can be related to each other through the time-independent Bogolyubov coefficients, $\alpha_{k}$ and $\beta_{k}$, as

$$
\underline{v}_{k}(\tilde{\eta})=\alpha_{k} v_{k}(\tilde{\eta})+\beta_{k} v_{k}^{*}(\tilde{\eta}) .
$$

The Bogolyubov coefficients satisfy the relation $\left|\alpha_{k}\right|^{2}-\left|\beta_{k}\right|^{2}=1$ via the condition in Equation (49). Comparing Equations (69) and (52), it follows that the creation and annihilation operators associated with two families of mode functions (i.e, those with and without 'underline') are related as

$$
\hat{a}_{\mathbf{k}}=\alpha_{k} \underline{\hat{a}}_{\mathbf{k}}+\beta_{k}^{*} \hat{\underline{a}}_{\mathbf{k}}^{\dagger} .
$$

Working in the Heisenberg picture, an initial vacuum state of the system, say $|\tilde{0}\rangle$ connected to the 'underlined' modes, $\underline{v}_{k}(\tilde{\eta})$, is the vacuum state of the system for all times. Then, the number operator, $\hat{N}_{\mathbf{k}}=\left(\hbar \ell^{3}\right)^{-1} \hat{a}_{\mathbf{k}}^{\dagger} \hat{a}_{\mathbf{k}}$, associated to the particles in $v_{k}$ mode, gives the average number of particles in the $|\tilde{0}\rangle$ vacuum. Thus, the $v_{k}$-mode-related vacuum state contains

$$
\mathcal{N}_{k}:=\left\langle\tilde{0}\left|\hat{N}_{\mathbf{k}}\right| \tilde{0}\right\rangle=\left|\beta_{k}\right|^{2},
$$

particles in the $\underline{v}_{k}$-mode vacuum.

Let us rewrite the mode solution, $\underline{v}_{k}$, in the WKB approximation from Equation (55), as ${ }^{4}$

$$
\underline{v}_{k}(\tilde{\eta})=\frac{1}{\sqrt{W_{k}(\tilde{\eta})}}\left[\alpha_{k} e_{k}(\tilde{\eta})+\beta_{k} e_{k}^{*}(\tilde{\eta})\right]
$$


where

$$
e_{k}(\tilde{\eta}):=\exp \left(-i \int^{\tilde{\eta}} d \eta W_{k}(\eta)\right) .
$$

Taking the time $(\tilde{\eta})$ derivative of $\underline{v}_{k}(\tilde{\eta})$, we obtain

$$
\underline{v}_{k}^{\prime}(\tilde{\eta})=-i \sqrt{W_{k}}\left[\alpha_{k} e_{k}(\tilde{\eta})-\beta_{k} e_{k}^{*}(\tilde{\eta})\right] .
$$

Inverting Equations (72) and (74) yields a relation for $\beta_{k}$ as

$$
\beta_{k}=\frac{\sqrt{W_{k}}}{2}\left(\underline{v}_{k}-\frac{i}{W_{k}} \underline{v}_{k}^{\prime}\right) e_{k} .
$$

Since the initial condition in LQC is fixed at the quantum bounce, $\tilde{\eta}=\tilde{\eta}_{b}$, by setting $\alpha_{k}=1$ and $\beta_{k}=0$ at $\tilde{\eta}=\tilde{\eta}_{b}$, we assume that no particle is created at the bounce. This yields the following conditions on $\underline{v}_{k}$ :

$$
\underline{v}_{k}\left(\tilde{\eta}_{b}\right)=1 / W_{k}\left(\tilde{\eta}_{b}\right) \text { and } \underline{v}_{k}^{\prime}\left(\tilde{\eta}_{b}\right)=-i W_{k}\left(\tilde{\eta}_{b}\right) \underline{v}_{k}\left(\tilde{\eta}_{b}\right),
$$

where $e_{k}\left(\tilde{\eta}_{b}\right)=1$. Then, for any time $\tilde{\eta}>\tilde{\eta}_{b}$, the number of particles produced becomes

$$
\mathcal{N}_{k}(\tilde{\eta})=\frac{1}{4}\left(W_{k}\left|v_{k}\right|^{2}+W_{k}^{-1}\left|v_{k}^{\prime}\right|^{2}-2\right) .
$$

Here, for convenience, we have dropped the 'underline' for the mode functions after the bounce $\left(\tilde{\eta}>\tilde{\eta}_{b}\right)$, i.e., we assume $\underline{v}_{k} \equiv \underline{v}_{k}\left(\tilde{\eta}_{b}\right)$ and $v_{k}(\tilde{\eta}) \equiv \underline{v}_{k}(\tilde{\eta})$ for $\tilde{\eta}>\tilde{\eta}_{b}$.

Having the number of particles produced per mode, $\mathcal{N}_{k}(\tilde{\eta})$, at a given time $\tilde{\eta}>\tilde{\eta}_{b}$, we can compute the total number density of production, $\mathcal{N}(\tilde{\eta})$, as the limit of $\sum_{k} \mathcal{N}_{k}(\tilde{\eta})$ in a box of volume $\ell^{3} \rightarrow \infty$, divided by the volume of the universe, $V=\ell^{3}\left|\tilde{a}_{1} \tilde{a}_{2} \tilde{a}_{3}\right|=\ell^{3} \tilde{c}^{3 / 2}$, as

$$
\mathcal{N}(\tilde{\eta})=\frac{\ell^{3}}{V} \sum_{k} \mathcal{N}_{k}(\tilde{\eta})=\frac{1}{4 \tilde{c}^{3 / 2}} \sum_{k}\left(W_{k}\left|v_{k}\right|^{2}+W_{k}^{-1}\left|v_{k}^{\prime}\right|^{2}-2\right) .
$$

The energy density of the created particles reads $\varrho_{k}(\tilde{\eta}) \equiv W_{k}(\tilde{\eta}) \mathcal{N}_{k}(\tilde{\eta})$ for each mode. Thus, the total energy density is obtained by summing the overall modes as

$$
\rho_{\mathrm{par}}=\frac{1}{\tilde{c}^{2}} \sum_{k} \varrho_{k}(\tilde{\eta}),
$$

where

$$
\varrho_{k}(\tilde{\eta})=\frac{1}{4}\left(\left|v_{k}^{\prime}\right|^{2}+W_{k}^{2}\left|v_{k}\right|^{2}-2 W_{k}\right) .
$$

At the bounce, $\tilde{\eta}=\tilde{\eta}_{b}$, the energy density of production is zero, $\varrho_{k}\left(\tilde{\eta}_{b}\right)=0$, as expected. However, for $\tilde{\eta}>\tilde{\eta}_{b}$, particles will be produced as the universe expands. In the following, we will analyze the energy density of production in the assumed adiabatic regime.

Following the (adiabatic) regularization scheme, the energy density of created particles is obtained as

$$
\rho_{\mathrm{par}}^{(\mathrm{ren})}=\frac{1}{\tilde{c}^{2}} \sum_{k}\left(\varrho_{k}\left[v_{k}(\tilde{\eta})\right]-\underline{\varrho_{k}}(\tilde{\eta})\right),
$$

where

$$
\begin{aligned}
\underline{\varrho_{k}}(\tilde{\eta}) & =\frac{1}{16}\left[\frac{\left(\tilde{\omega}_{k, \tilde{\eta}}^{\prime}\right)^{2}}{\tilde{\omega}_{k, \tilde{\eta}}^{3}}+\tilde{\omega}_{k, \tilde{\eta}}\left(\epsilon_{2}^{(2)}\right)^{2}-\frac{\left(\tilde{\omega}_{k, \tilde{\eta}}^{\prime}\right)^{2}}{2 \tilde{\omega}_{k, \tilde{\eta}}^{3}} \epsilon_{2}^{(2)}+\frac{\tilde{\omega}_{k, \tilde{\eta}}^{\prime}}{\tilde{\omega}_{k, \tilde{\eta}}^{2}} \epsilon_{2}^{(3)}\right] \\
& =\varrho_{k}^{(0)}+\varrho_{k}^{(2)}+\varrho_{k}^{(4)} .
\end{aligned}
$$


It turns out that $\varrho_{k}$ does not fall off faster than $k^{-4}$ when $k \rightarrow \infty$. The zeroth adiabatic order term in Equation (82) is zero, $\varrho_{k}^{(0)}=0$, and the divergences are included in the second and fourth-order terms (for massive modes). For the massless modes, the divergences are included only in the fourth-order term. Therefore, the renormalized energy density of created particles, Equation (81), can be obtained by subtracting the adiabatic vacuum energy of the particle productions up to the fourth order. When considering the higher order terms (more than the fourth order) in the adiabatic mode functions, $v_{k}(\tilde{\eta})$, associated to the vacuum state $|0\rangle$, particles are produced and the total energy density of the created particles is proportional to $1 / \tilde{c}^{2}(\tilde{\eta})$. For an isotropic case (either massive or massless), where $\tilde{c}=\tilde{a}^{2}$, we get $\rho_{\text {par }}^{(\text {ren })} \propto 1 / \tilde{a}^{4}(\tilde{\eta})$, which scales as radiation. This is a similar situation derived in [22]. Therefore, unlike the classical FLRW cosmology, even massless modes contain nonzero particle production due to quantum gravity effects.

In the standard classical cosmology, the WKB ansatz yields a divergent asymptotic series in the adiabatic parameter so that the particle production phenomenon is associated with the violation of the WKB approximation or the region where the WKB approximation is not fulfilled very well [34]. However, in the present setting, since the spacetime region transitions between the super-Planckian and sub-Planckian regimes, where the nature of the background geometry differs from the original classical isotropic spacetime to an anisotropic dressed geometry due to quantum gravity effects, the changes in the vacuum and creation of particles are inevitable.

In the pre-inflationary scenario considered in [22], by assuming a massless field in an isotropic quantum cosmological background, $\tilde{a}(\tilde{\eta})=\tilde{c}^{1 / 2}(\tilde{\eta})$, an integration range was chosen in Equation (79) by the window of observable modes of the CMB. Therein, it has been argued that when taking a UV cutoff at the characteristic momentum $k_{b}=k_{*}\left(\tilde{\eta}_{b}\right)=$ $\left.\sqrt{\tilde{a}^{\prime \prime}(\tilde{\eta}) / \tilde{a}(\tilde{\eta})}\right|_{\tilde{\eta}_{b}} \approx 3.21 m_{\mathrm{Pl}}$ and an infra-red (IR) cutoff at $k_{*}(\tilde{\eta})=\sqrt{\tilde{a}^{\prime \prime}(\tilde{\eta}) / \tilde{a}(\tilde{\eta})}=\lambda_{*}^{-1}$ for all $\tilde{\eta}>\tilde{\eta}_{b}$, being the physical energy of particles after they reenter the effective horizon, the energy density of created particles becomes

$$
\rho_{\text {par }}=0.012 m_{\mathrm{Pl}}^{4} / \tilde{a}^{4}(\tilde{\eta}) .
$$

This gives the energy density of particles produced in the region $k_{*} \leq k \leq k_{b}$. Thus, the main contribution to the production of particles is the modes whose wavelengths, $\lambda$, hold the range $\lambda_{b} \leq \lambda \leq \lambda_{*}$, i.e., the modes that reenter $\lambda_{*}$ after the bounce during the pre-inflationary phase and will only reexit $\lambda_{*}$ again in the slow-roll inflationary phase.

This implies that the energy density of particles produced in the quantum gravity regime, which scales as relativistic matter, is significant comparing to the background energy density. The background energy density during the slow-roll inflation is dominant over the backreaction of particle productions. However, it should be guaranteed that the production density is still dominant in the pre-inflationary phase before beginning the slow-roll phase. By assuming that the elapsed e-foldings between the bounce and the onset of inflation is about 4-5 e-folds, the energy density of the backreaction is smaller than the background energy density as $\rho_{\text {par }} \lesssim\left\langle\hat{\rho}_{\varphi}\right\rangle \approx 2 \times 10^{-5} \mathrm{~m}_{\mathrm{Pl}}^{4}$. However, the analysis in [22] indicates that the energy density (Equation (83)) is two orders of magnitude larger than the required upper bound, $2 \times 10^{-5} \mathrm{~m}_{\mathrm{Pl}}^{4}$, as estimated for the density of particle creation during the pre-inflationary phase. It turns out that the backreaction of produced particles cannot be neglected so that a more careful analysis of the backreacted wave function for the background quantum geometry is needed.

The above argument indicates that when starting from Equation (27), the backreaction of the field modes on the quantum background is not negligible; therefore, the total wave function, $\Psi\left(v, Q_{\mathbf{k}}, \phi\right)$, cannot be decomposed as $\Psi=\Psi_{o} \otimes \psi$. It may even make a further constraint on expanding the right-hand-side of Equation (27) to derive the evolution Equation (28). Nevertheless, if we suppose that the approximation in Equation (28) is valid, the presence of the backreaction would lead to a modification of the total state as $\Psi=\Psi_{o} \otimes \psi+\delta \Psi$ (cf. [18,20]). Taking into account such modification, the dispersion 
relation of the field, propagating on the emergent effective geometry, will be modified such that the local Lorentz symmetry becomes violated. In such a scenario, each mode feels a distinctive background geometry, which depends on that mode; a rainbow dressed background emerges. On such backgrounds, the standard approach for studying the infinite number of field modes fails, and an alternative procedure should be employed (e.g., see [35]). For other approaches in canonical quantum gravity, where the quantum theory of cosmological perturbations and their backreactions are implemented, see, e.g., [36-42].

\section{Conclusions and Discussion}

In this paper, the quantum theory of an (inhomogeneous) massive test field, $\varphi$, propagating on a quantized FLRW geometry is addressed. The background geometry constitutes of a homogeneous massless scalar field, $\phi$, as matter source, which plays the role of internal time in quantum theory. From an effective point of view, due to quantum gravity effects on the background geometry, quantum modes of the field can experience a dressed spacetime whose geometry differs from the original FLRW metric. If the backreaction of the field modes, $Q_{\mathbf{k}}$, on the background is discarded, within a test field approximation, the full quantum state of the system can be decomposed as $\Psi\left(v, Q_{\mathbf{k}}, \phi\right)=\Psi_{o}(v, \phi) \otimes \psi\left(Q_{\mathbf{k}}, \phi\right) ; \Psi_{o}$ and $\psi$ are the quantum states of the (unperturbed) background and the field modes, respectively.

In the interaction picture, an evolution equation emerges for $\psi\left(Q_{\mathbf{k}}, \phi\right)$, which resembles the Schrödinger equation for the same field modes propagating on a time-dependent dressed spacetime, whose metric components are functions of the quantum fluctuations of the FLRW geometry. For massive and massless modes, there exists a wide class of solutions for the effective dressed background metric. The massless modes can only experience an isotropic and homogeneous dressed background with a dressed scale factor, $\tilde{a}(\phi)$ (cf. see [13]). The massive modes, however, yield a general class of solutions for the emergent dressed geometries that resembles the anisotropic Biachi I spacetimes. Likewise, the scale factors $\left(\tilde{a}_{1}, \tilde{a}_{2}, \tilde{a}_{3}\right)$ of the consequent dressed Bianchi-I metric are functions of fluctuations of the isotropic quantum geometry.

Given a dressed anisotropic spacetime, as a solution discussed above, we reviewed the standard quantum field theory on such a background. More precisely, we investigated the issue of gravitational particle production associated with the field modes on the dressed Bianchi I geometry in a suitably chosen adiabatic regime. This led to some backreaction issues in the super-Planckian regime, which may affect the dynamics of the early universe. To have a regularized energy-momentum operator of the test field, the adiabatic vacuum state was chosen up to fourth-order terms. We computed the energy density of the particle production within this adiabatic limit. The divergences in the energy density of the produced particles were regularized within the fourth-order adiabatic terms and the remaining terms change as $\rho_{\mathrm{par}}^{\text {(ren) }} \propto 1 /\left(\tilde{a}_{1} \tilde{a}_{2} \tilde{a}_{3}\right)^{4 / 3}$. Some phenomenological issues related to such particle production were discussed. It was demonstrated that the backreaction due to the particle production in the super-Planckina regime may have significant effects on the evolution of the universe and may subsequently modify the existing pre-inflationary scenario of LQC [16] (cf. [22]).

Funding: This paper is based upon work from the European Cooperation in Science and Technology (COST) action CA18108-Quantum gravity phenomenology in the multi-messenger approach supported by COST.

Acknowledgments: The results of the present review article are mainly based on my past collaborations with J. C. Fabris and S. Rastgoo.

Conflicts of Interest: The authors declare no conflict of interest. 


\section{Notes}

$1 \quad$ We consider an elementary cell $\mathcal{V}$ by fixing its edge to lie along the coordinates $\left(\ell_{1}, \ell_{2}, \ell_{3}\right)$. We then denote the volume of $\mathcal{V}$ by $\dot{V}=\ell_{1} \ell_{2} \ell_{3} \equiv \ell^{3}$, and restrict all integrations in the Fourier integral to this volume.

2 Note that our aim at the moment is just to avoid the UV divergence terms when regularizing the energy-momentum of the field. However, to have a more complete mode solution, in particular, when exploring the issues of the particle productions or backreaction effects, as we will see later, higher order terms in $W_{k}(\tilde{\eta})$ should be taken into account.

3 They satisfy the normalization condition (Equation (49)) for the mode functions.

4 It should be noticed that the standard WKB ansatz may yield a divergent asymptotic series in the adiabatic parameter. Thus, when investigating the particle production in a time-dependent background, an optimal number of terms in that series should be chosen so that the resulting truncated WKB series becomes exponentially small. Such precision would still be insufficient to describe particle production from vacuum. Therefore, an adequately precise approximation should be employed by improving the WKB ansatz [34].

\section{References}

1. Birrell, N.D.; Davies, P.C.W. Quantum Fields in Curved Space; Cambridge University Press: Cambridge, UK, 1984.

2. Parker, L.E.; Toms, D. Quantum Field Theory in Curved Spacetime; Cambridge Monographs on Mathematical Physics; Cambridge University Press: Cambridge, UK, 2009. [CrossRef]

3. Hu, B.L.; Parker, L. Anisotropy Damping Through Quantum Effects in the Early Universe. Phys. Rev. 1978, D17, 933-945; Erratum: Phys. Rev. D 1978, 17, 3292. [CrossRef]

4. Zeldovich, Y.B. Particle production in cosmology. Pisma Zh. Eksp. Teor. Fiz. 1970, 12, 443-447.

5. Ashtekar, A.; Bojowald, M.; Lewandowski, J. Mathematical structure of loop quantum cosmology. Adv. Theor. Math. Phys. 2003, 7, 233-268. [CrossRef]

6. Ashtekar, A.; Lewandowski, J. Background independent quantum gravity: A Status report. Class. Quant. Grav. 2004, 21, R53, [CrossRef]

7. Rovelli, C. Quantum Gravity; Cambridge Monographs on Mathematical Physics; Cambridge University Press: Cambridge, UK, 2004. [CrossRef]

8. Thiemann, T. Modern Canonical Quantum General Relativity; Cambridge Monographs on Mathematical Physics; Cambridge University Press: Cambridge, UK, 2007. [CrossRef]

9. Ashtekar, A. Loop Quantum Cosmology: An Overview. Gen. Rel. Grav. 2009, 41, 707-741. [CrossRef]

10. Ashtekar, A.; Pawlowski, T.; Singh, P. Quantum Nature of the Big Bang: An Analytical and Numerical Investigation. I. Phys. Rev. 2006, D73, 124038, [CrossRef]

11. Ashtekar, A.; Wilson-Ewing, E. Loop quantum cosmology of Bianchi I models. Phys. Rev. 2009, D79, 083535, [CrossRef]

12. Ashtekar, A.; Singh, P. Loop Quantum Cosmology: A Status Report. Class. Quant. Grav. 2011, 28, 213001, [CrossRef]

13. Ashtekar, A.; Kaminski, W.; Lewandowski, J. Quantum field theory on a cosmological, quantum space-time. Phys. Rev. 2009, D79, 064030, [CrossRef]

14. Agullo, I.; Ashtekar, A.; Nelson, W. Extension of the quantum theory of cosmological perturbations to the Planck era. Phys. Rev. 2013, D87, 043507, [CrossRef]

15. Agullo, I.; Ashtekar, A.; Nelson, W. The pre-inflationary dynamics of loop quantum cosmology: Confronting quantum gravity with observations. Class. Quant. Grav. 2013, 30, 085014, [CrossRef]

16. Agullo, I.; Ashtekar, A.; Nelson, W. A Quantum Gravity Extension of the Inflationary Scenario. Phys. Rev. Lett. 2012, 109, 251301, [CrossRef] [PubMed]

17. Ashtekar, A.; Barrau, A. Loop quantum cosmology: From pre-inflationary dynamics to observations. Class. Quant. Grav. 2015, 32, 234001, [CrossRef]

18. Dapor, A.; Lewandowski, J.; Tavakoli, Y. Lorentz Symmetry in QFT on Quantum Bianchi I Space-Time. Phys. Rev. 2012, D86, 064013, [CrossRef]

19. Dapor, A.; Lewandowski, J. Metric emerging to massive modes in quantum cosmological space-times. Phys. Rev. 2013, D87, 063512, [CrossRef]

20. Lewandowski, J.; Nouri-Zonoz, M.; Parvizi, A.; Tavakoli, Y. Quantum theory of electromagnetic fields in a cosmological quantum spacetime. Phys. Rev. D 2017, 96, 106007, [CrossRef]

21. Tavakoli, Y.; Fabris, J.C. Creation of particles in a cyclic universe driven by loop quantum cosmology. Int. J. Mod. Phys. 2015, D24, 1550062, [CrossRef]

22. Graef, L.L.; Ramos, R.O.; Vicente, G.S. Gravitational particle production in loop quantum cosmology. Phys. Rev. D 2020, 102, 043518, [CrossRef]

23. Garcia-Chung, A.; Mertens, J.B.; Rastgoo, S.; Tavakoli, Y.; Vargas Moniz, P. Propagation of quantum gravity-modified gravitational waves on a classical FLRW spacetime. Phys. Rev. D 2021, 103, 084053, [CrossRef]

24. Rastgoo, S.; Tavakoli, Y.; Fabris, J.C. Phenomenology of a massive quantum field in a cosmological quantum spacetime. Ann. Phys. 2020, 415, 168110, [CrossRef]

25. Lankinen, J.; Vilja, I. Gravitational Particle Creation in a Stiff Matter Dominated Universe. JCAP 2017, 08, 025, [CrossRef] 
26. Scardua, A.; Guimarães, L.F.; Pinto-Neto, N.; Vicente, G.S. Fermion Production in Bouncing Cosmologies. Phys. Rev. D 2018, 98, 083505, [CrossRef]

27. Assanioussi, M.; Dapor, A.; Lewandowski, J. Rainbow metric from quantum gravity. Phys. Lett. 2015, B751, 302-305. [CrossRef]

28. Celani, D.C.F.; Pinto-Neto, N.; Vitenti, S.D.P. Particle Creation in Bouncing Cosmologies. Phys. Rev. D 2017, 95, 023523, [CrossRef]

29. Bolliet, B.; Barrau, A.; Grain, J.; Schander, S. Observational exclusion of a consistent loop quantum cosmology scenario. Phys. Rev. D 2016, 93, 124011, [CrossRef]

30. Ashtekar, A.; Pawlowski, T.; Singh, P. Quantum nature of the big bang. Phys. Rev. Lett. 2006, 96, 141301, [CrossRef]

31. Parker, L.; Fulling, S.A. Adiabatic regularization of the energy momentum tensor of a quantized field in homogeneous spaces. Phys. Rev. 1974, D9, 341-354. [CrossRef]

32. Chakraborty, B. The mathematical problem of reflection solved by an extension of the WKB method. J. Math. Phys. 1973, 14, 188. [CrossRef]

33. Fulling, S.A.; Parker, L.; Hu, B.L. Conformal energy-momentum tensor in curved spacetime: Adiabatic regularization and renormalization. Phys. Rev. 1974, D10, 3905-3924. [CrossRef]

34. Winitzki, S. Cosmological particle production and the precision of the WKB approximation. Phys. Rev. D 2005, 72, 104011, [CrossRef]

35. Martin, J.; Brandenberger, R.H. The TransPlanckian problem of inflationary cosmology. Phys. Rev. D 2001, 63, 123501, [CrossRef]

36. Bojowald, M.; Ding, D. Canonical description of cosmological backreaction. J. Cosmol. Astropart. Phys. 2021, 03, 083, [CrossRef]

37. Fernández-Méndez, M.; Mena Marugán, G.A.; Olmedo, J. Hybrid quantization of an inflationary model: The flat case. Phys. Rev. D 2013, 88, 044013, [CrossRef]

38. Gomar, L.C.; Fernández-Méndez, M.; Marugán, G.A.M.; Olmedo, J. Cosmological perturbations in Hybrid Loop Quantum Cosmology: Mukhanov-Sasaki variables. Phys. Rev. D 2014, 90, 064015, [CrossRef]

39. Gomar, L.C.; Martín-Benito, M.; Marugán, G.A.M. Gauge-Invariant Perturbations in Hybrid Quantum Cosmology. J. Cosmol. Astropart. Phys. 2015, 06, 045, [CrossRef]

40. Bojowald, M.; Hossain, G.M.; Kagan, M.; Shankaranarayanan, S. Anomaly freedom in perturbative loop quantum gravity. Phys. Rev. D 2008, 78, 063547, [CrossRef]

41. Cailleteau, T.; Barrau, A.; Grain, J.; Vidotto, F. Consistency of holonomy-corrected scalar, vector and tensor perturbations in Loop Quantum Cosmology. Phys. Rev. D 2012, 86, 087301, [CrossRef]

42. Cailleteau, T.; Mielczarek, J.; Barrau, A.; Grain, J. Anomaly-free scalar perturbations with holonomy corrections in loop quantum cosmology. Class. Quant. Grav. 2012, 29, 095010, [CrossRef] 